\title{
PASOS PARA LA PLANIFICACIÓN DE UNA INVESTIGACIÓN CLÍNICA
}

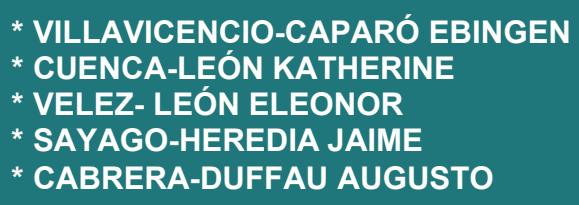

Recibido: $15 / 11 / 2015$

Aceptado: 18/11/2015

\section{RESUMEN}

El objetivo del presente artículo fue hacer una revisión bibliográfica de las recomendaciones para realizar una investigación científica clínica. Se presenta aspectos de conformación del equipo, definición de la pregunta de investigación, identificar la variable de interés, marco teórico, diseño del estudio, tamaño de muestra, el análisis estadístico, recursos necesarios para el estudio, aspectos bioéticos de la investigación, reclutamiento de pacientes, toma de datos y la estrategias de publicación de los resultados.

Palabras Clave: Estudio Clínico, Proyectos de Investigación, Metodología.

\section{ABSTRACT}

The aim of this paper was to make a literature review of the recommendations for clinical research. It shows aspects of setting up the team, defining the research question, identify the variable of interest, theoretical framework, study design, sample size , statistical analysis, resources needed for the study, bioethical aspects of research, recruitment is presented patients, data and strategies for the results publication.

Keywords: Clinical Study, Research Design, Methodology.

\section{I.- INTRODUCCIÓN}

La sección denominada Tool box, tiene como propósito dar herramientas que permitan ser una referencia rápida para las personas que se inician en el proceso de la investigación científica en ciencias de la salud.

En esta oportunidad revisaremos los pasos que se recomiendan para la planificación de una investigación clínica.

\section{II.-ESTADO DEL ARTE ${ }^{1,2,3}$ \\ CONFORMACIÓN DEL EQUIPO}

Toda investigación implica la orquestación sincronizada de varias competencias, en este texto proponemos como mínimo 3 participantes en el estudio. El primero es el experto especialista en el contenido del tema a investigar, el segundo es el metodólogo-bioestadístico y el tercero es el observador-evaluador, quien se calibrará para la toma de los datos.

Antes del inicio de la investigación se debe hacer un plan de publicación del estudio, indicando quien es el responsable de redactar cada una de las partes del artículo, de igual forma se debe dejar en claro si la autoría es compartida o existe un autor y los demás son colaboradores y hasta el orden en el que aparecerán los nombres de los autores en la publicación final, de tal manera que se eviten conflictos y logremos un equipo colaborador donde todos sepan que les corresponde.

\section{DEFINIR LA PREGUNTA DE INVESTIGACIÓN}

Tenemos 5 tipos de preguntas de investigación, las cuales son respecto a:

- Factores de riesgo para las enfermedades.

- Frecuencia de las enfermedades.

- Valor de los métodos de diagnóstico.

- Efectividad de las terápias.

- Pronóstico de las enfermedades. 
Para este efecto algunas universidades preconizan la estrategia de usar el acrónimo $\mathrm{PICO}^{3}$, donde "P" significa población, la "I" se refiere a la intervención que se desea evaluar, la "C" viene de la intervención con la que se va a comparar y la "O" viene de "outcome" o resultado que se va a evaluar.

\section{IDENTIFICAR LA VARIABLE DE INTERES}

Otro paso importante en la planificación es conocer realmente la variable de resultado clínico relevante y diferenciarla de las variables intermedias o variables que nos ayudan a construir la variable final.

Por ejemplo la medición de profundidad de sondaje periodontal, es una variable intermedia, la variables de resultados clínicos relevantes serán la movilidad dentaria, el dolor, la funcionalidad para masticar. Es decir variables que tengan relevancia para el paciente.

\section{MARCO TEÓRICO}

Conocer la teoría al respecto nos permite tomar una posición respecto al tema y también nos habilita para analizar, explicar y predecir lo que pensamos encontrar con nuestra investigación.

Dentro de la construcción del marco teórico talvez encontramos que existen bases de datos que ya contienen la información necesaria para responder la pregunta de investigación, o para tener la línea base sin la necesidad de conseguir data nueva. Algunos estudios en las universidades debieran tener archivos de bases de datos, que puedan permitir hacer seguimiento a pacientes o evaluar el progreso de la enfermedad en ciertas poblaciones.

\section{EL DISEÑO DEL ESTUDIO 4}

La elección del diseño del estudio está muy ligado al tipo de pregunta de investigación, así tenemos que para:

Factores de riesgos el diseño es caso control.

Frecuencia de enfermedades el diseño es transversal.

Valor de métodos diagnóstico, el diseño es de sensibilidad y especificidad.

Efectividad de las terapias, el diseño es ensayo clínico aleatorizado.

Pronóstico de enfermedades, el diseño es cohortes.

De acuerdo a lo que la ciencia conoce de la enfermedad nos permite plantear una investigación que nos lleve a producir el mayor nivel de evidencia, en las investigaciones clínicas los niveles de acuerdo a su jerarquía son como se muestra a continuación*:

\section{NIVELES DE EVIDENCIA CLÍNICA*}

1a Revisión sistemática de ensayos clínicos aleatorizados.

1b Ensayo clínico aleatorizado ECA (con intervalo de confianza pequeño)

2a Revisión sistemática de estudios de cohortes

$2 \mathrm{~b}$ Estudio de cohorte o ECA con bajo porcentaje de seguimiento $<80 \%$

3a Revisión sistemática de estudios de casos-controles

3b Estudio de caso-control

4 Estudio de serie de casos o estudio de cohorte o estudio de caso control de baja calidad.

5 Opinión de experto o reporte de un caso

*Journal of Evidence-Based Dental Practice

\section{TAMAÑO DE MUESTRA ${ }^{5}$ (¿cuántas personas debo evaluar?)}

Debemos tener en cuenta que hay dos vías para el cálculo del tamaño muestral:

1.- En estudios Observacionales, donde se pretende calcular el tamaño de una sola muestra, debemos utilizar la fórmula para estimar la cantidad mínima de personas que necesito evaluar para que se exprese la enfermedad en las proporciones que normalmente existe en la población total.

2.- En los estudios Experimentales o comparativos, donde se pretende calcular el tamaño de dos muestras para compararlas, es necesario saber que hay una diferencia conceptual importante entre significancia estadística y significancia clínica. Porque podemos encontrar estudios que demuestren diferencia estadísticamente significativa, sin embargo esta diferencia no alcanza a ser clínicamente relevante. En ese caso la primera pregunta que se debe plantear el investigador es: cuál es el tamaño más pequeño, en la variable respuesta, que sea clínicamente relevante. Y a continuación se necesita saber qué tamaño de efecto sugieren 
los estudios previos. En este caso el tamaño de muestra se basa en la comprobación de una hipótesis, por lo tanto se toma en cuenta la variabilidad de la variable, el tamaño del efecto y la magnitud del error que nos permitimos, al comprobar la hipótesis (error tipo I o a asociado a la confiabilidad del estudio y error tipo II o $\beta$ asociado a la potencia del estudio) siendo valores usuales para a 0.05 y 0.01 ; y para $\beta 0.8$ y 0.9 .

\section{LA VARIABILIDAD ESPERADA EN LA VARIABLE RESPUESTA ${ }^{6}$}

Cualquiera de las dos vías del cálculo del tamaño de muestra, incluye en su fórmula la variabilidad esperada, esto implica conocer el comportamiento estadístico de la variable, mediante estudios previos. En tal sentido influyen a esta variabilidad la precisión del instrumento, la variabilidad biológica y la variabilidad en otros aspectos del contexto. Si no se tiene el dato de la variabilidad de la variable se puede recurrir a la prueba piloto para obtener este valor y lograr un tamaño de muestra adecuado.

\section{EL ANÁLISIS ESTADÍSTICO QUE SE UTILIZARÁ}

En términos generales la comprobación de hipótesis puede caer en una de estas tres opciones:

a) Comparación de promedios (T de Student, ANOVA, otros)

b) Comparación de proporciones (Chi cuadrado, otros)

c) Correlación de variables (R2 de Pearson, regresión lineal, regresión múltiple)

Adicionalmente debemos planificar la mejor manera de presentar los resultados tanto en tablas como en gráficos, para que el clínico los pueda entender de una manera rápida y fácil. Otra implicancia del análisis estadístico es que está muy relacionado con el cálculo del tamaño de muestra.

\section{RECURSOS NECESARIOS PARA EL ESTUDIO.}

Hacer la estimación de los recursos humanos necesarios para llevar a cabo un estudio clínico, va a depender de la cantidad de procesos y pacientes que existan, se necesita saber, acerca de las actividades a realizar, entre ellas la movilización de los pacientes, de los investigadores y del personal auxiliar. No olvidar de incluir estimación de tiempo y recursos para el manejo y soporte bioestadístico.Muchas veces se tiene en cuenta solamente los costos directos del estudio, sin embargo existen costos indirectos que también deben de ser considerados al momento de calcular el presupuesto de la investigación. Algunas veces los estudios que son ganadores de una subvención, no tienen cubiertos los gastos indirectos y si no se tiene una estrategia para solventar este gasto es posible que la investigación quede trunca.

\section{ASPECTOS BIOÉTICOS DE LA INVESTIGACIÓN}

Toda investigación realizada en personas o animales, deben de pasar por la revisión de un comité institucional de ética, que garantice la protección de los mismos. En el caso de estudios con humanos además se debe de implementar un consentimiento informado. Muchas veces estos comités toman algunas semanas en dar el visto bueno, se debe considerar este tiempo en la planificación del estudio. Los estudios que son subvencionados exigen pasar el protocolo por un comité de ética.

\section{RECLUTAMIENTO/ INSCRIPCIÓN / RETENCIÓN DE LAS UNIDADES DE ESTUDIO Y TOMA DE DATOS}

Se debe tener claro el mecanismo de reclutamiento de pacientes para el estudio, es decir dónde los voy a encontrar, de igual forma tenemos que tener en claro la cantidad de pacientes que podemos enrolar en el estudio en un período de tiempo. De manera práctica, se le pregunta al clínico ¿Cuántos pacientes cree ud. que puede reclutar para este estudio, en el tiempo determinado?

Antes de empezar el estudio es muy necesario tener un manual de organización y funciones que describe en detalle que hará cada uno de los participantes y en qué momento. Tan detallado debe estar el manual que inclusive contempla la posibilidad de que uno o varios de los miembros del grupo de clínicos, puede ser reemplazado por otro profesional con iguales capacidades. Este manual debe también indicar que acciones tomar en caso de efectos adverso o situaciones de crisis.

\section{PLAN PARA COMPARTIR LOS DATOS CON OTROS INVESTIGADORES}

Aunque suene extraño muchos investigadores ponen a disposición del público sus bases de datos, debido a que frecuentemente no se logra utilizar al $100 \%$ todas las posibilidades de exploración de resultados en una base de datos. Una vez publicados los resultados principales del estudio, no tiene sentido guardar información tan valiosa, conviene encontrar personas dispuestas a hacer análisis de datos y generar más investigaciones aprovechando un estudio clínico bien realizado, a esto se le denomina minería de datos (DATA MINING). 


\section{III.- CONCLUSIONES}

Hacer una investigación clínica es una oportunidad para lograr conocimientos valiosos en bien de la humanidad. En tal sentido el diseño del estudio debe tener la rigurosidad que nos permita extrapolar los resultados a la mayor cantidad posible de personas.

La observación cuidadosa de las etapas previas al inicio de la toma de datos (planificación del estudio) nos garantiza llevar adelante un trabajo que tendrá buenos resultados, independientemente de la comprobación o rechazo de la hipótesis planteada.

La investigación clínica es una actividad de un equipo de investigadores, casi siempre con tres componentes, el investigador principal, un asesor temático y un asesor metodológico o estadístico.

El correcto planteamiento del tamaño de muestra y la elección bien meditada de las pruebas estadísticas para la comprobación de la hipótesis, nos conducirán a evitar el error tipo I y error tipo II.

La observancia de los aspectos bioéticos es fundamental para la publicación válida de investigaciones científicas, sin embargo también la ética en la relación entre los integrantes del equipo de investigación, es otro pilar fundamental de la gestión de la investigación.

El conocer la normativa vigente, en el país o países que se desarrollará la investigación es importante para el correcto desarrollo del estudio, tanto en el campo clínico como en el desarrollo de tecnología de apoyo al diagnóstico y tratamiento.

Finalmente tener una agenda de fuentes de cooperación para la investigación o de instituciones públicas o privadas que ofrecen financiamiento para la investigación es también una tarea importante y de primer orden para el planteamiento y planificación de una investigación clínica.

\section{REFERENCIAS BIBLIOGRÁFICAS}

1.- Lesaffre E.; Feine J.; Leroux B.; Declerck D. Statistical and Methodological Aspects of Oral Health Research. Edit. John Wiley \& Sons Ltd. United Kingdom 2009.

2.- Forrest JL, Miller SA. Evidence-Based Decision Making in Action: Part 1 - Finding the Best Clinical Evidence. J Contemp Dent Pract 2002 August;(3)3: 010-026.

3.- Hernández-Sampieri, R. Metodología de la Investigación científica. 5ta Edición. México, D.F.: McGraw Hill, 2010. [ISBN 978-607-15-0291-9]

4.- Hernández-Avila M, Garrido-Latorre F, López-Moreno S. Diseño de estudios epidemiológicos. Salud Pública México [Internet]. abril de 2000 [citado 8 de marzo de 2016];42(2):144-54. Recuperado a partir de: http://www.scielosp.org/scielo.php?script=sci abstract\&pid=S0036-

$36342000000200010 \&$ Ing=en\&nrm=iso\&tlng=es

5.-Villavicencio-Caparó E. El tamaño muestral en tesis de post grado ¿cuántas personas debo encuestar? Research Gate. [Online]. Arequipa; 2010 [Visitado 01 -enero-2016 Disponible en: https://www.researchgate.net/publication/283352423 EL TAMANO MUESTRAL EN TESIS DE POST GRADO CUANTAS PERSONAS DEBO ENCUESTAR.

6.-Rosner B. Fundamentals of Biostatistics. Edit.Cengage Learning; U.S.A. $\quad 2010.896$ p.

7. Damián-Navarro, L; Flores-Mori, M; Flores-Mena, B.El Consentimiento Informado en Odontología, un Análisis Teórico. Revista Estomatológica Herediana, vol. 24, núm. 1, enero-marzo, 2014, pp. 42-47 